\title{
Protocol
}

\section{The effect of post colonoscopy abdominal massage on abdominal pain, distension, discomfort and patient satisfaction: a randomized controlled study protocol}

\author{
Dilek Ozturk $^{1 *}$, Aysel Gurkan ${ }^{2}$ \\ ${ }^{1}$ Marmara University Health Sciences Institute, Istanbul, Turkey \\ ${ }^{2}$ Department of Nursing, Marmara University Faculty of Health Sciences, Istanbul, Turkey
}

Received: 03 June 2021

Revised: 12 July 2021

Accepted: 14 July 2021

\author{
*Correspondence: \\ Dilek Ozturk, \\ E-mail: dilekozturk20@ hotmail.com
}

Copyright: $\odot$ the author(s), publisher and licensee Medip Academy. This is an open-access article distributed under the terms of the Creative Commons Attribution Non-Commercial License, which permits unrestricted non-commercial use, distribution, and reproduction in any medium, provided the original work is properly cited.

\begin{abstract}
Background: Abdominal pain, distension, and discomfort are the most frequently seen side effects after colonoscopy. These side effects can cause physical and mental results and increased workloads and care costs. Additionally, the satisfaction of the patients with the colonoscopy process may affect their willingness to undergo repeated colonoscopies in the future which may be required for the surveillance of colon malignity. Currently, there are no studies evaluating the effects of abdominal massage on post colonoscopy these abdominal symptoms. The aim of the study was to evaluate the effect of post colonoscopy abdominal massage on abdominal pain, distension, discomfort, and patient satisfaction.

Methods: This study was designed as a prospective randomized controlled study. Participants meeting inclusion criteria will be assigned to intervention and control groups using a random number generator. Participants assigned to the intervention group will receive abdominal massages twice a day after colonoscopy, and participants assigned to the control group will receive only 'routine care'. The abdominal pain, distension, discomfort, and satisfaction levels of the patients will be evaluated using the Visual analogue scale.

Conclusions: New approaches are needed to improve the physiological and psychological health levels of patients after colonoscopy and help them return to their daily life activities and social lives faster. The trial will provide valuable evidence to inform clinical application and help evaluate the effects of the use of the relevant intervention.

Trial registration: Clinical trials registration number NCT04979351.
\end{abstract}

Keywords: Colonoscopy, Abdominal massage, Abdominal pain, Abdominal distension, Abdominal discomfort, Patient satisfaction

\section{INTRODUCTION}

Abdominal pain, bloating, and discomfort are the most frequently problems experienced by patients undergoing colonoscopy. ${ }^{1-6}$ These problems are thought to be caused by several factors such as the duration of the process, the technical difficulty level of the process, distension caused by air insufflation, and the expertise level of the endoscopist. ${ }^{4,7}$ These problems are experienced by approximately a third of patients undergoing colonoscopy, and may last from a few hours to a few days. ${ }^{3}$ In a recent study, $44 \%$ of patients were found to experience such minor side effects 24 hours after colonoscopy, and $23 \%$ were found to experience them after a 30 day follow-up. ${ }^{1}$

Although these problems are dubbed minor side effects, they may cause serious results for patients with regard to physical comfort and mental stress and may necessitate patients to be monitored post colonoscopy and followed up closely. ${ }^{1,2,4}$ This may lead to an increase in costs by increasing the length of hospital stay and nursing care requirements. ${ }^{8}$ Additionally, the satisfaction of the patients with the colonoscopy process may affect their 
willingness to undergo repeated colonoscopies in the future which may be required for the surveillance of colon malignity. ${ }^{5}$

For these reasons, decreasing post colonoscopy abdominal pain, distension, and discomfort and increasing patient satisfaction may increase the acceptance of future colonoscopy screening for colorectal cancers. $^{9}$

Studies focused on decreasing abdominal symptoms after colonoscopy and increasing patient comfort and satisfaction have yielded varying results. For example, while some studies have reported that rectal tube application after colonoscopy reduces pain and increases patient comfort and satisfaction, in another study, it was determined that rectal tube application post colonoscopy had no effect on abdominal distension, pain, discomfort, and patient satisfaction after the process and 24 hours later. ${ }^{10,11}$

In another study, rectal aspiration applied right after colonoscopy was found to be more effective in decreasing abdominal symptoms compared to the application of rectal tubes after colonoscopy. ${ }^{5}$ In another study where total colonic decompression through repeating cecal intubation after colonoscopy was applied, the method was found to decrease the distension complaints of patients in the early post colonoscopy period from $59 \%$ to $25 \%$. However, in this study, no significant difference between the groups that did and did not receive decompression with regard to abdominal pain and distension could be found 24 to 48 hours after the procedure. $^{12}$

In yet another study, no significant difference with regard to pain intensity could be found between the aspiration of air in the curves of the colon without repeated intubation while the colonoscope was being taken out and rectal aspiration. ${ }^{8}$

Abdominal massaging has been shown to help improve symptoms in patients with severe constipation. ${ }^{13}$ In the literature, it has been reported that abdominal massage relaxes the abdominal muscles and stimulates the excretory activity by supporting the intestine, decrease discomfort and pain in individuals with abdominal pain related to cramps or flatulence, and increase the quality of life. ${ }^{13,14-18}$ Moreover, it is a non-invasive and safe nonpharmacological method. It has no known side effects and does not require any cost. ${ }^{15,16,19}$

We assumed that abdominal massaging could improve post colonoscopy abdominal symptoms. However, to date, there are no studies evaluating the effects of abdominal massaging on post colonoscopy abdominal symptoms.

Therefore, we designed a prospective study to evaluate the aspect of post colonoscopy abdominal massage that concerns endoscopy nurses, namely, the effect on abdominal pain, distension, discomfort, and patient satisfaction.

\section{Aim}

The aim of the study was to evaluate the effect of post colonoscopy abdominal massage on abdominal pain, distension, discomfort, and patient satisfaction.

\section{Null hypothesis}

There will be no significant difference between patients receiving abdominal massage post colonoscopy and those not receiving such an intervention with regard to abdominal pain, distension, discomfort, and patient satisfaction.

\section{METHODS}

\section{Trial design}

In this trial, a two-group design where each group is assigned an equal number of random participants will be used. This design will enable examine the effect of the intervention on the outcome variables.

\section{Setting}

The trial will be conducted in the endoscopy unit of a third level public hospital in Istanbul, Turkey.

\section{Participants and sampling}

The minimum sample size was calculated using power analysis in the $\mathrm{G}^{*}$ power (v3.1.7) program. In the calculation performed by taking the post application pain measurements in the previous study [mean $8.3(\mathrm{SD}=15.7)$ for rectal tube; and mean $2.9(\mathrm{SD}=8.6)$ for rectal aspiration] into consideration and seeking a significance level of $0.05(\alpha)$ and a statistical testing power of $80 \%$ (1- $\beta$ ) the effect size was calculated to be (d) 0.427 and according to the standard deviation value (SD) the necessary sample size was found to be 176 ( $\mathrm{N}=88$ for each group). ${ }^{5}$ It was decided to include a total of 180 patients in the study and assign 90 to the intervention group and 90 to the control group.

Inclusion criteria were defined as receiving outpatient non-sedated colonoscopy for diagnosis and scanning, being of 18 years of age and above, owning a cell phone, having a body mass index below 30 , being conscious and having location, people, and time orientation, having no communication barriers, being able to communicate in Turkish, and giving written informed consent to participate in the study.

Exclusion criteria were receiving sedation, having previously received intestine resection or other intestinal surgery, having an uncontrolled psychopathological illness, receiving colonoscopy for treatment, and having active lower gastrointestinal system bleeding. Removal 
criteria were colonoscopy remaining unfinished because of insufficient bowel cleaning, desiring to leave the study of one's own volition, and not complying with study processes and conditions.

\section{Randomization}

The participants will be selected from the endoscopy appointment list. The randomization of patients who meet inclusion criteria will be performed through a random number generator.

\section{Instruments}

\section{The demographic and clinical information form}

This form, which was prepared by the researchers based on the literature, included the demographic characteristics [age, sex, height, weight, body mass index (BMI), marital status, education level, occupation, employment status, income level, health insurance, smoking, alcohol use, additional systemic diseases, medication used continuously, history of colorectal cancer in the family, excretion habits] of the patients and their clinical characteristics (history of colonoscopy, whether the patient was informed regarding colonoscopy, current colonoscopy indication, whether the patient experienced an unwanted event during intestinal preparation, colonoscopy placement depth, the start and finish times of colonoscopy, and process duration) as well as their phone numbers for communication. ${ }^{5,11,20}$

\section{The visual analog scale (VAS)}

VAS, which is a single dimension scale widely used for the measurement of subjective parameters, consists of emotional adjectives in which each emotion experienced by the person at that moment is marked with a vertical line on a plane. ${ }^{21-24}$

VAS, an extremely simple, effective, repeatable, measuring tool that requires minimal tools, consist of a $10 \mathrm{~cm}$ long line vertically or horizontally. The two extreme descriptors of the subjective category are placed on two ends of this line $(0=$ 'no emotion at all', $10=$ 'the worst feeling imaginable'). The patient is asked to mark the line in the most appropriate location for the intensity of his/her feeling.

The length from the lowest VAS level to the marking of the patient is measured with a ruler, yielding the numeric index of the emotional intensity of the patient in centimeters and millimeters. ${ }^{24-27}$ A result of $0 \mathrm{~cm}$ indicates no emotion, 1 to $3.9 \mathrm{~cm}$ indicates 'mild' emotion, 4 to $5.9 \mathrm{~cm}$ indicates 'moderate' emotion, and 6 to $10 \mathrm{~cm}$ indicates 'strong' emotion. ${ }^{27}$

Although the horizontal and vertical forms of the VAS have been found to have good correlation, vertical use has been reported to cause an inclination to mark higher scores and horizontal use has been reported to increase clinical performance with less possibility of error. ${ }^{21,26-28}$ Thus, the horizontal VAS will be used in this study.

The reasons behind the selection of this scale in this trial to evaluate the post colonoscopy abdominal pain $(0=$ 'no pain', $10=$ 'unbearable pain'), distension $(0=$ 'no distension', $10=$ 'unbearable distension'), abdominal comfort $(0=$ 'not comfortable at all', $10=$ 'completely comfortable') and satisfaction ( $0=$ 'not satisfied', $10=$ 'completely satisfied') levels of the patients were this scale being tested for validity and reliability in our country and this scale being used in many other studies. $^{24,25,29}$

\section{The monitoring forms}

The post colonoscopy abdominal symptoms levels of the control group patients after the process (day 0) at the unit and at home and in the morning and evening of the $1^{\text {st }}$ day after the process will be recorded on this form, as well as the post colonoscopy abdominal symptoms of the intervention group patients after the process (day 0) and in the morning and evening of the first day before and after the application of abdominal massage.

Additionally, the satisfaction levels of both groups at the end of the $1^{\text {st }}$ day will be recorded.

\section{Intervention}

The participants will be assigned to one of the two groups according to the randomization list. Participants assigned to the intervention group will receive abdominal massages twice a day after colonoscopy, and participants assigned to the control group will receive only 'routine care'.

In the trial, the abdominal massage application will be conducted according to the 'Abdominal massage application guide' developed by Uysal, Eşer, and Akpınar in our country (Table 1).

Accordingly, abdominal massaging has been suggested to be applied twice a day, 30 minutes after a meal for 15 minutes. The required tools for application are a towel and liquid vaseline. The massage movements include three basic maneuvers, namely effleurage, petrissage, and vibration. ${ }^{15,30-32}$ Before the application, the patient will be given a lying down position and the head of the bed will be raised by $30^{\circ}-45^{\circ}$.

After the abdominal area is unclothed with special attention to the privacy of the patient, the skin will be dried with a towel if it is moist. In order to ensure that the hands move easily across the skin of the patient and to avoid discomfort, liquid vaseline will be used. After pouring an amount of liquid vaseline to the palms and spreading to the hands according to the guide, the area will be massaged for 15 minutes. After the massage, the abdominal area will be covered and the patient will lie down in a comfortable position. ${ }^{19,30,33}$ 
Table 1: The abdominal massage application guide.

\begin{tabular}{|c|c|}
\hline 1. & The hands are washed. \\
\hline 2. & The patient and his/her next of kin are informed on the process. \\
\hline 3. & $\begin{array}{l}\text { Whether there is pain, sensitivity, tension, rashes, or disruptions in skin integrity in the abdominal area are } \\
\text { evaluated, as well as how full the bladder is. }\end{array}$ \\
\hline 4. & $\begin{array}{l}\text { The patient is given a lying down position and if there is risk involved, the head of the bed is raised by } 30-45 \\
\text { degrees. }\end{array}$ \\
\hline 5. & The abdominal area is unclothed with special attention to privacy. If the skin is moist, it is dried with a towel. \\
\hline 6. & $\begin{array}{l}\text { A small amount of liquid vaseline is poured into the massager's hands and the hands are rubbed together to } \\
\text { warm up the hands and distribute the vaseline. }\end{array}$ \\
\hline 7. & Abdominal massage is started. \\
\hline 8. & $\begin{array}{l}\text { The massage begins with surface effleurage. The abdominal region is rubbed down from the upper epigastric } \\
\text { region, over the iliac bones (the point where the femur meets the vertebral column), and to the groin from both } \\
\text { sides of the pelvis with mild pressure. This prevents reactionary abdominal wall tension to the first touch. }\end{array}$ \\
\hline 9. & $\begin{array}{l}\text { After the abdominal wall relaxes; } \\
\text { - In a manner to ensure all motions are clockwise } \\
\text { Taking the anatomical abdominal area starting from the anterior superior side of the right iliac bone, } \\
\text { following the costa bone line, to the anterior superior side of the left iliac bone into consideration, } \\
\text { First, the lower right side of the abdominal area where the ascending colon is located and the upper right } \\
\text { side of the abdominal area is massaged, going to the upper left side (for the transverse colon) and the lower } \\
\text { left sides (for the descending colon), following the rib line. } \\
\text { Each quadrant must be massaged last at least } 1 \text { minute and apply with moderate pressure. }\end{array}$ \\
\hline 10. & $\begin{array}{l}\text { After rubbing, petrissage is applied with the palm in the same order of quadrants. } \\
\text { - After rubbing, the right hand is placed on the lower right corner of the abdominal area. After supporting with } \\
\text { the left hand, the hands make circles in } 4-5 \mathrm{~cm} \text { diameters, and the pertisage is applied clockwise (the } \\
\text { procession direction of the intestines). }\end{array}$ \\
\hline 11. & Following rubbing and petrissage movements are repeated for 15 minutes. \\
\hline 12. & $\begin{array}{l}\text { The speed of the massage movements and the number of repetitions should be on a normal level. The pressure } \\
\text { and strength of the application should be arranged according to the sensitivity of the patient. }\end{array}$ \\
\hline 13. & $\begin{array}{l}\text { A vibrating motion is given using the fingertips for a minute at the end, and the procedure is ended with a } \\
\text { rubbing motion. }\end{array}$ \\
\hline 14. & The patient is covered and given a comfortable position. \\
\hline 15. & The hands are washed. \\
\hline
\end{tabular}

\section{Intervention group}

The first face to face interview with the patients in this group will be conducted by the first author of the study an hour before colonoscopy. In this interview, the 'Demographic and clinical information form' will be filled out and abdominal massage training will be provided. Additionally, patients and their next of kin will be informed on the use of VAS and how to record abdominal pain, distension, discomfort, and satisfaction levels.

The second face to face interview will be conducted after colonoscopy in the monitoring room. In this interview, the abdominal pain, distension, and discomfort levels of the patients will be evaluated using the VAS and recorded on the monitoring form (T0). Follow up measurements will be performed at 7 different time points. The first abdominal massage will be applied by the researcher and 30 minutes after the completion of the massage, the abdominal pain, distension, and discomfort levels of the patients will be evaluated using the VAS and recorded (T1). Before discharge from the unit, the patients and their next of kin will be given explanations on the subjects not understood regarding the abdominal massage application, VAS, and the use of the monitoring form. Additionally, the patients and their next of kin will be given the 'Abdominal massage application guide', a VAS for each measurement time point, and the 'Monitoring form'.

The second abdominal massage application will be performed at home by the patient or his/her next of kin at the evening of the day the colonoscopy was performed (day 0). The third and fourth abdominal massages will be performed the $1^{\text {st }}$ day after colonoscopy in the morning and evening by patients or their next of kin.

Abdominal pain, distension, and discomfort levels will be measured using VAS before each application (T2, T4, and T6) and 30 minutes after (T3, T5, and T7) each application and recorded on the monitoring form. Additionally, satisfaction levels will be recorded on the monitoring form at the end of the $1^{\text {st }}$ day after colonoscopy. Phone communication will be established with patients or their next of kin in the evening of the 
colonoscopy to support massage training and at the end of the $1^{\text {st }}$ day to receive the screenshot of the monitoring form.

\section{Control group}

The first face to face interview with the patients in this group will be conducted by the first author of the study an hour before colonoscopy. In this interview, the 'Demographic and clinical information form' will be filled out. Additionally, patients and their next of kin will be informed on the use of VAS and how to record abdominal pain, distension, discomfort, and satisfaction levels.

The second face to face interview will be conducted after colonoscopy in the monitoring room. In this interview, 45 minutes after the colonoscopy, the abdominal pain, distension, and discomfort levels of the patients will be evaluated using the VAS and recorded on the monitoring form (T0). Before discharge from the unit, the patients and their next of kin will be given the VAS and the Monitoring Form, and explanations on the subjects not understood regarding the use of those tools will be given. The follow up measurements of the participants in this group will be performed in 3 different time points. Abdominal pain, distension, and discomfort levels will be measured with VAS and recorded on the monitoring form the evening of the colonoscopy (day 0) (T1), and in the morning (T2) and evening (T3) of the day after colonoscopy. Additionally, satisfaction levels will be recorded on the monitoring form at the end of the $1^{\text {st }}$ day after colonoscopy. Phone communication will be established with patients or their next of kin in the evening of the colonoscopy to support the patients and at the end of the $1^{\text {st }}$ day to receive the screenshot of the monitoring form (Figure 1).

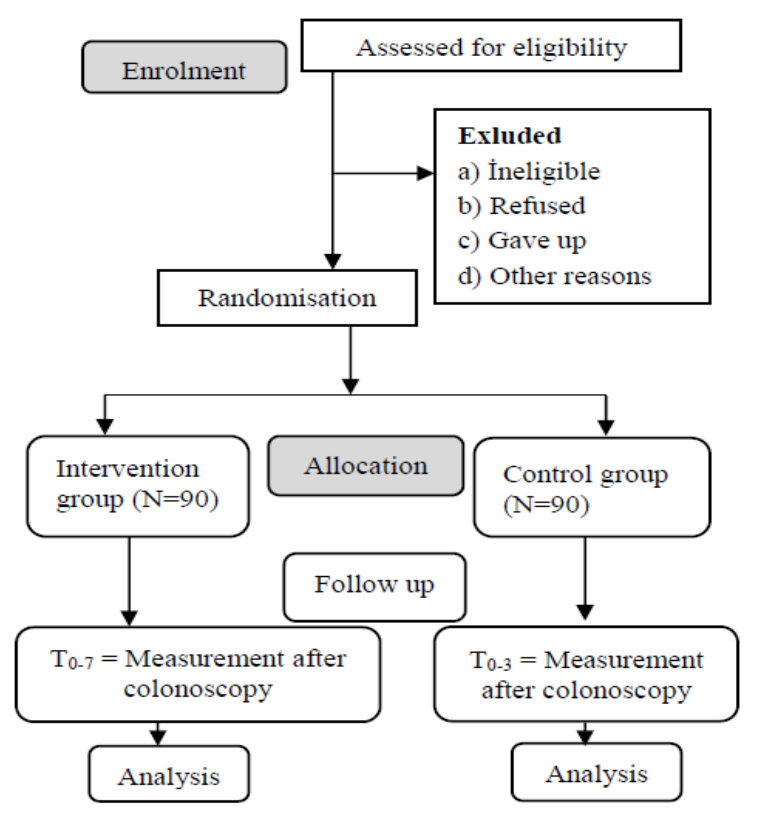

Figure 1: Study flow diagram.

\section{Ethical considerations}

This trial was approved by the Marmara University School of Medicine Ethics Board for Clinical Studies with the date 24.07.2020 and protocol code 09.2020.885. Additionally, permission was taken from the administration of the Prof. Dr. Cemil Taşcıoğlu City Hospital attached to the Istanbul Department of Health (Date:13.07.2020) where the study will be conducted and the Medical Specialty Education Board (Date:29.07.2020). Before data collection, the type and aim of the study will be explained to the participants as well as the application process and their rights regarding this process, and written informed consent will be sought. The principles of the 2008 Helsinki Declaration will be upheld throughout the study.

\section{Statistical analysis}

In the evaluation of the data obtained from the trial, the NCSS (Number Cruncher Statistical System) 2007 Statistical Software (NCSS LLC, Kaysville, Utah, USA) program will be used for statistical analyses. Beside descriptive statistical methods for data evaluation (mean values, standard deviation, median values, frequencies and rates), the Shapiro Wilks test and Box plot graphs will be used to evaluate the compliance of data with normal distribution. The intergroup evaluations of normally distributed variables will be performed using the Student t test, and the intergroup evaluations of data not complying with normal distribution will be performed using the Mann Whitney U test.

According to follow up measurements, the Repeated measures test and post-hoc Bonferroni test will be used in the intragroup evaluation of normally distributed variables, and the evaluations between two follow up measurements will be performed using the paired samples test. Intragroup follow ups not exhibiting normal distribution will be evaluated using the Friedman test and the post hoc Bonferroni corrected Dunn test. The relationships between variables will be evaluated using correlation analyses according to distribution, and advanced evaluations will be performed using Linear regression modeling. Qualitative data will be compared using the Pearson Chi squared test and the Fisher's exact test. The results will be evaluated using a $95 \%$ confidence interval and a statistical significance level of $\mathrm{p}<0.05$.

\section{DISCUSSION}

This trial is the first study meticulously evaluating the aspects of post colonoscopy abdominal massaging that concern endoscopy nurses; which are its effects on abdominal pain, distension, distension, and patient satisfaction.

Additionally, the study design will provide valuable evidence to inform clinical application and help to evaluate the effects of the use of the relevant intervention. 


\section{CONCLUSION}

Although colonoscopy is a generally well tolerated, safe diagnosis and treatment method it causes some side effects. It is noticeable that the frequently experienced effects of side effects such as abdominal pain, distention and discomfort on the patient after colonoscopy. The study will provide valuable evidence to endoscopy nurses by evaluating the effects of abdominal massage on patients' abdominal pain, distention, comfort and satisfaction after colonoscopy. It will also have positive effects in terms of the efficient use of the workforce by healthcare professionals.

\section{Funding: No funding sources}

Conflict of interest: None declared

Ethical approval: This trial was approved by the Marmara University School of Medicine Ethics Board for Clinical Studies and protocol code 09.2020.885. Additionally, permission was taken from the administration of the Prof. Dr. Cemil Taşcioğlu City Hospital attached to the Istanbul Department of Health where the study will be conducted and the Medical Specialty Education Board

\section{REFERENCES}

1. Steffenssen MW, Najami I, Nielsen E, Baatrup G. Patient-reported complications related to colonoscopy: a prospective feasibility study of an email-based survey. Acta Oncol. 2019;58(1):65-70.

2. Sumanac K, Zealley I, Fox BM, Rawlinson J, Salena B, Marshall JK, et al. Minimizing postcolonoscopy abdominal pain by using $\mathrm{CO}(2)$ insufflation: a prospective, randomized, double blind, controlled trial evaluating a new commercially available $\mathrm{CO}(2)$ delivery system. Gastrointest Endosc. 2002;56(2):190-4.

3. Erstad DJ, Krowsoski LS, Kaafarani HM. Abdominal Pain After Colonoscopy. Gastroenterology. 2017;152(3):486-7.

4. Yi CH, Liu TT, Lei WY, Hung JS, Chen CL. Influence of Rectal Decompression on Abdominal Symptoms and Anorectal Physiology following Colonoscopy in Healthy Adults. Gastroenterol Res Pract. 2016;4101248.

5. Liu TT, Yi CH, Lei WY, Yu HC, Hung JS, Chen CL. Comparison of rectal suction versus rectal tube insertion for reducing abdominal symptoms immediately after unsedated colonoscopy. Endosc Int Open. 2016;4(6):725-9.

6. Devitt J, Shellman L, Gardner K, Nichols LW. Using positioning after a colonoscopy for patient comfort management. Gastroenterol Nurs. 2011;34(2):93-100.

7. Kim SY, Kim HS, Park HJ. Adverse events related to colonoscopy: Global trends and future challenges. World J Gastroenterol. 2019;25(2):190-204.

8. Ozkan N, Kayaoglu HA, Ersoy OF, Çelik A. The effect of desufflation points on the pain cause by insufflated air during colonoscopy. Turkey Clinical J Med Sci. 2007;27:672-6.

9. Lynch I, Hayes A, Buffum MD, Conners EE. Insufflation using carbon dioxide versus room air during colonoscopy: Comparison of patient comfort, recovery time, and nursing resources. Gastroenterol Nurs. 2015;38(3):211-7.

10. Steinberg EN, Howden CW. Randomized controlled trial of rectal tube placement for the management of abdominal distension following colonoscopy. Gastrointest Endosc. 1997;46(5):444-6.

11. Hilzenrat N, Fich A, Odes HS, Krugliak P, Eidelman L, Gaspar N, et al. Does insertion of a rectal tube after colonoscopy reduce patient discomfort and improve satisfaction?. Gastrointest Endosc. 2003;57(1):54-7.

12. Lee JG, Vigil $H$, Leung JW. A randomized controlled trial of total colonic decompression after colonoscopy to improve patient comfort. Am J Gastroenterol. 2001;96(1):95-100.

13. Lamas K, Lindholm L, Stenlund H, Engstrom B, Jacobsson C. Effects of abdominal massage in management of constipation - A randomized controlled trial. Int J Nurs Stud. 2009;46(6):759-67.

14. Liu Z, Sakakibara R, Odaka T, Uchiyama T, Yamamoto T, Ito T, et al. Mechanism of abdominal massage for difficult defecation in a patient with myelopathy (HAM/TSP). J Neurol. 2005;252(10):1280-2.

15. Turan N, Astı TA. The importance of abdominal massage in management of constipation. J Anatolia Nurs Health Sci. 2015;18(2):148-54.

16. McClurg D, Hagen S, Dickinson L. Abdominal massage for the treatment of constipation (protocol). Cochrane. 2011;4.

17. Sinclair M. The use of abdominal massage to treat chronic constipation. J Bodyw Mov Ther. 2011;15(4):436-45.

18. Dehghan M, Fatehi Poor A, Mehdipoor R, Ahmadinejad M. Does abdominal massage improve gastrointestinal functions of intensive care patients with an endotracheal tube?: A randomized clinical trial. Complement Ther Clin Pract. 2018;30:122-8.

19. McClurg D, Lowe-Strong A. Does abdominal massage relieve constipation? Nurs Times. 2011;107(12):20-2.

20. Arabul M, Kandemır A, Çelık M, Alper E, Akpinar $\mathrm{Z}$, Aslan $\mathrm{F}$, et al. Impact of an information video before colonoscopy on patient satisfaction and anxiety. Turk J Gastroenterol. 2012;23(5):523-9.

21. Aygin D, Var G. Pain management in trauma patients and nursing approaches. Sakarya Med J. 2012;2(2):61-70.

22. Çetinkaya F, Karabulut N. The impact on the level of anxiety and pain of the training before operation given to adult patients who will have abdominal operation. J Anatolia Nurs Health Sci. 2010;13(2):20-6.

23. Chou R, Gordon DB, Casasola OA, Rosenberg JM, Bickler S, Brennan $\mathrm{T}$, et al. Management of 
Postoperative Pain: A Clinical Practice Guideline From the American Pain Society, the American Society of Regional Anesthesia and Pain Medicine, and the American Society of Anesthesiologists' Committee on Regional Anesthesia, Executive Committee, and Administrative Council. J Pain. 2016;17(2):131-57.

24. Aydin A, Araz A, Asan A. Visual analog scale and affect grid: An application to Turkish Culture. Turkish Psychol. 2011;14(27):1-13.

25. Ayan M, Taş U, Söğüt E, Arıcı S, Karaman S, Esen M, Demirtürk F. Comparing efficiencies of diclofenac sodium and paracetamol in patients with primary dysmenorrhea pain by using Visual Analog Scale. Agri. 2013;25(2):78-82.

26. Gallagher EJ, Liebman M, Bijur PE. Prospective validation of clinically important changes in pain severity measured on a visual analog scale. Ann Emerg Med. 2001;38(6):633-8.

27. Kjeldsen HB, Klausen TW, Rosenberg J. Preferred Presentation of the Visual Analog Scale for Measurement of Postoperative Pain. Pain Pract. 2016;16(8):980-4.

28. Gagliese L, Weizblit N, Ellis W, Chan VWS. The measurement of postoperative pain: A comparison of intensity scales in younger and older surgical patients. Pain. 2005;117(3):412-20.
29. Aslan F. Visual comparison and comparison of sensitivity and selectivity of simple descriptive scales in postoperative pain assessment. Nat Nurs Cong. 1998;178-86.

30. Uysal N, Eşer İ, Akpinar H. The effect of abdominal massage on gastric residual volume: A randomized controlled trial. Gastroenterol Nurs. 2012;35(2):117-23.

31. Olgun S. Abdominal massage application in management of constipation. Ege Uni Fac Nurs J. 2016;32(3):118-26.

32. Altun Ugras G, Yüksel S, Isik MT, Tasdelen B, Dogan H, Mutluay O. Effect of abdominal massage on bowel evacuation in neurosurgical intensive care patients. Nurs Crit Care. 2020:1-9.

33. Preece J. Introducing abdominal massage in palliative care for the relief of constipation. Complement Ther Nurs Midwifery. 2002;8(2):101-5.

Cite this article as: Ozturk D, Gurkan A. The effect of post colonoscopy abdominal massage on abdominal pain, distension, discomfort and patient satisfaction: a randomized controlled study protocol. Int J Clin Trials 2021;8(4):301-7. 